\title{
La Cité Universitaire Internationale, Ferdinand Brunot et la linguistique
}

\begin{abstract}
Anne COSTE
La Cité Universitaire Internationale, dont les travaux avaient débuté en 1923, fut inaugurée en 1925. Depuis, et ce jusqu'à ce jour, elle reçoit les étudiants étrangers venus poursuivre leurs études en France. A l'occasion du premier Congrès Mondial de Linguistique Française qui se tient dans ses murs, j'ai entrepris de rappeler dans quelles conditions elle fut créée : le rôle éminent joué par Ferdinand Brunot (FB désormais), le célèbre auteur de l'Histoire de la langue française, (HLF) donne à la rencontre de ce lieu et du Congrès Mondial de Linguistique Française une résonance particulière.

La vie de FB est foisonnante, les documents qui en portent le témoignage sont nombreux. J'ai relevé ceux qui semblent indiquer comment $\mathrm{FB}$ en vient à conclure à la nécessité de créer une Cité qui accueillerait les étudiants étrangers.
\end{abstract}

\section{La formation de F. Brunot}

FB est né le 6 novembre 1860 à Saint-Dié dans les Vosges. Il fait de brillantes études chez les Frères de la Doctrine chrétienne et est reçu Bachelier ès Lettres à Nancy en 1876. Il passe ensuite un an en Allemagne, séjour qui n'est certainement pas sans incidence pour l'avenir, comme il l'évoque beaucoup plus tard, en 1909, dans son discours au Congrès des Professeurs de langues vivantes : «C'est que, il y a presque trente ans, j'ai prêché moi-même l'exemple, en commençant - chose rare à l'époque - mes études de philologie par un séjour à l'étranger ». Il est reçu à l'Ecole Normale Supérieure le 8 août 1879 au vingt-troisième rang et il est licencié ès Lettres en 1880, cacique de l'agrégation de grammaire en 1882.

Comme tout normalien, il commence sa carrière dans l'enseignement en lycée, à Bar-le-Duc. Il n'y reste qu'une année, du 6 octobre 1882 au 4 octobre 1883. Son directeur écrit dans son unique rapport, le 3 juillet 1883 : « Il réussit dans l'enseignement secondaire, mais il sera prochainement réclamé avant peu par l'enseignement supérieur ». FB a effectivement postulé sur un poste de maître de conférences à la Faculté des Lettres de Nancy. Finalement, il est nommé maître de conférences de langues et littératures anciennes à la Faculté des Lettres de Lyon le 24 juillet 1883. Il y sévira jusqu'au $1^{\text {er }}$ décembre 1891. C'est à Lyon que FB pose ses premières marques personnelles : il aborde la vie politique - en 1890 il est président de l'Union patriotique du Rhône - et surtout, en 1887 il publie son Précis de Grammaire historique de la langue française qui reçoit un prix de l'Académie. Il y il réalise une étude de la langue française en dehors du latin, premier ancrage grammatical officiel et remarqué : «A Lyon (il) enseignait le grec et le latin et cherchait et trouvait déjà sa voie dans la fondation d'une discipline nouvelle : l'histoire de la langue française », G. Cohen, Le Temps, 4 février 1938.

Le 25 juin 1891, FB est reçu Docteur ès Lettres, à l'unanimité. Il soutient sa thèse avec les deux sujets :

1- De Philibert Bugnoni vita et eroticis versibus

2- La doctrine de Malherbe, d'après son commentaire sur Desportes

Le premier représente l'attrait d'une reconnaissance officielle d'une école de poésie qui ne s'apparente ni à l'Ecole de Ronsard ni à celle de Marot mais peut-être à celle de l'Ecole Lyonnaise. Cette thèse et celle sur Malherbe montrent l'intérêt que porte FB pour l'histoire de la langue française. On peut y voir les prémisses de l'HLF.

Par un arrêté du 19 novembre 1891, FB est nommé maître de conférences de grammaire et de philologie à la Faculté des Lettres de Paris. Par un arrêté du 13 novembre 1900, il est élevé au rang de professeur dans 
une chaire d'Histoire de la langue française qui est créée par le même décret (la nomination de FB est actée dans l'article premier).

Désormais, FB se pose en défenseur de la langue française : il combat pour une réforme de l'orthographe, de l'agrégation, de l'enseignement de la grammaire, combattant la Grammaire de l'Académie, etc. Cette défense prend au fil des années sa caractéristique propre autour de deux idées directrices : (1) la victoire du français sur le latin, (2) l'unification de la France à partir de la langue. Mariangela Roselli, dans sa thèse « La langue française entre science et République, 1880-1950 », 1994, remarque que FB représente le « miroir des enjeux de la langue dans la nation ».

\section{L'œuvre scientifique}

L'activité de recherche scientifique et pédagogique de FB comprend des publications et une participation active aux décisions du Ministère de l'Instruction publique.

Au nombre de ses livres :

- Le Précis de grammaire historique

1887 ,

- L'Histoire de la langue française

à partir de

1905 ,

- La Méthode de langue française

1909 ,

- La Pensée et la Langue

Institutionnellement, on retiendra :

- la réforme de l'orthographe et celle de l'enseignement de la grammaire

- la création des Archives de la Parole

Les liens sont nombreux entre la réflexion générale de La Pensée et la langue et les préceptes pédagogiques de la Méthode de langue française_écrite en collaboration avec N. Bony. Dans ce manuel destiné à l'enseignement primaire, l'enfant apprend comment traduire les causes, le doute, la volonté, le désir, la certitude, éléments qui apparaîtront dans La Pensée et la langue sous la rubrique des «faits de pensée ». Autre exemple, cité par N. Bony dans l'introduction de la Méthode: "Notre livre ne présente plus par exemple un chapitre : DE L'ADJECTIF, mais un chapitre DE LA QUALIFICATION où l'on apprend à exprimer la qualité des êtres et des choses, leurs divers degrés. ॥

FB exprime lui-même dans l'introduction de La Pensée et la langue :

«Dès 1903, ma conviction était faite, et dans les Méthodes élémentaires que j'ai publiées à cette époque, en collaboration avec M. Bony,(...) j'ai fait des applications timides encore, du système, en réunissant tous les moyens d'expression qui servent à marquer les causes, les buts, les conséquences, les hypothèses, etc. Dans le cours de méthodologie, que je professais alors à la Sorbonne, 1908, je marquais fortement la nécessité d'abandonner les 'parties du discours'. »

L'idée développée par FB consiste à « dresser des méthodes de langage, où les faits ne soient plus rangés d'après l'ordre des signes, mais d'après l'ordre des idées. " La Pensée et la langue, Introduction, pXX. FB destine cet ouvrage de neuf cents pages qui demande une réflexion nouvelle sur la langue, qui n'est pas un recueil de règles grammaticales et qui ne s'adresse à un niveau défini d'étude, aux « spécialistes destinés à propager la connaissance dans le monde entier, je leur aurais donné une méthode libératrice. »

Et FB a rencontré un écho certain si l'on en juge par le courrier provoqué par La Pensée et la langue déposé au Fonds Brunot de la bibliothèque de l'Institut, en provenance de la France et de l'étranger. Voici quelques exemples : 


\section{1 de France}

- H. Guénot, 19 juin 1922, exprime son plaisir «à comprendre » ces faits de pensée «considérés par rapport au langage ».

- M. Juret, de Strasbourg, 2 mars 1925, écrit avoir utilisé la méthode avec succès : «J'ai la satisfaction de voir que les faits viennent d'eux-mêmes ».

- Mme Missard, ancienne Sévrienne et professeur au lycée de Caen, 22 février 1923, dit trouver dans ce livre la justification de sa lutte contre l'incompétence de sa directrice et pouvoir poursuivre ainsi son travail pédagogique et grammatical avec plus d'assurance.

\section{2 de l'étranger}

- M. Brandin, lettre du 19 juin 1922 écrit à FB : «Vous pouvez compter sur moi pour les (vos idées) propager ». Dans sa lettre du 8 décembre suivant il annonce qu'il va publier un compte rendu dans Modern Languages.

- M. Hanse écrit dans sa bibliographie grammaticale : «Ce lourd volume devrait se trouver dans toutes les bibliothèques de nos établissements d'instruction »

- Le Pr. Fuchs, 19 novembre et 15 décembre 1922, demande à FB d'obtenir «à moindre prix » cet ouvrage pour la bibliothèque de son université à Berlin.

FB affirme lui-même dans l'introduction de la troisième édition, 1935 :

«L'un d'entre eux (des grammairiens) m'a fait l'honneur de me dire qu'avec la méthode que je préconise, on peut établir la théorie d'une langue gheez ancien éthiopien, dont il est impossible de ramener les faits aux cadres de la grammaire ordinaire. »

Il s'agit probablement de Sylvain Grébaut qui enseigne l'éthiopien en tant que professeur à l'Institut Catholique de Paris à partir de 1926. Déjà, dans une lettre du 23 novembre 1922, il déclare réfléchir à des applications possibles aux langues sémitiques « qui seront extrêmement intéressantes ».

$\mathrm{FB}$, en toute circonstance de sa vie professionnelle, reste très attentif aux réactions de ces "spécialistes ", français et étrangers, qui se manifestent aussi lors du débat concernant la réforme de l'orthographe, (1903-1906) auquel FB prend part activement. Quelles sont ses motivations?

\subsubsection{La référence en matière orthographique est l'Académie, cela irrite FB :}

«Ce que l'Académie défend ce n'est pas son œuvre, c'est le patrimoine commun des français, la langue nationale ».

Par ailleurs, quelle est l'orthographe défendue par l'Académie ? Celle

«à laquelle on prétend réserver le titre ronflant d'orthographe nationale est, en vérité, une greffe étrangère, une des formes de l'écorcherie du latin qui corrompt et défigure notre langue depuis si longtemps »

«Seulement l'orthographe est-elle la langue ? » demande FB (p. 21 de sa Lettre ouverte à Monsieur le Ministre sur la Réforme de l'Orthographe), et d'ajouter

" L'écriture est un signe. Changer quelque chose au signe n'est pas, sauf peut-être dans quelques cas tout à fait exceptionnels, changer quelque chose à la chose signifiée. »

\subsubsection{L'orthographe constitue un problème à l'école primaire :}

« ... considérez comme il en va de l'école primaire, consultez sur ce point, Monsieur le Ministre, tous ceux qui se passionnent pour l'enseignement 
national, à défaut de $\mathrm{M}$ Gréard mort, que l'Académie ne voulut point entendre, demandez à vos directeurs, à vos inspecteurs : le cri sera unanime. L'orthographe est le fléau de l'école. »

Pourquoi ? Parce que, l'enseignement de l'orthographe française ne peut se fonder sur la raison mais sur la mémoire visuelle et la répétition mécanique et devient « abêtissant, déformateur et contre éducatif ».

FB retrouve cette même difficulté chez les enseignants du français, langue étrangère :

«Pendant les dix ans que j'ai eu l'honneur de diriger les cours d'étrangers à l'Alliance française, j'ai eu cinq à six mille étrangers, à peu près tous professeurs de français. Je n'ai jamais entendu une voix discordante. Tous se plaignaient d'être inutilement empêtrés dans un fourré de difficultés orthographiques où les élèves perdaient leur temps et leur peine. »

Aussi,
«Comment répandre dans les classes populaires en France et à l'étranger une langue hérissée de difficultés orthographiques, héritée de latinisants et cercles et salons et académies de notables? ».
J.-Cl. Chevalier, Langages, Larousse, juin 1994, p61

Le grand principe selon FB pour réformer l'orthographe, tel qu'il l'énonce dans son rapport de la deuxième commission (p. 17) :

«Un signe unique pour chaque son, un son unique pour chaque signe, c'est le phonétisme ».

Et il développe :

«L'art souverain serait ici dans l'adaptation rigoureuse du signe à la chose signifiée, dans l'harmonie parfaite entre le son et la lettre, car aucune impression de beauté ne peut se comparer à la jouissance esthétique si haute et si pure que donne la contemplation d'une fin entièrement réalisée par un moyen d'une idéale simplicité. »"

Pour FB le mot « beau » est « le mot simple, sobre, uni et dépouillé ».

Ce principe est favorablement accueilli par les professeurs de français comme A. Thomas, partisan de «l'anarchie simplificatrice» (lettre du 25 octobre 1906), plus encore par des maîtres des pays non francophones: les Etats-Unis (première réponse), l'Argentine, la Bulgarie, la Hollande... Voici ce qu'écrit le 17 août 1906 à FB Lucien Abeille, professeur de français en Argentine et membre de la Société de Linguistique de Paris :

«Il faut enseigner le français à l'étranger pour se rendre compte de la portée immense de la simplification pour l'expansion de la langue française (...) Nos élèves n'arrivent jamais à connaître l'orthographe, qu'ils détestent d'ailleurs. S'il vous était agréable de recevoir quelques dictées je vous les enverrais et vous vous convaincriez qu'elles sont écrites naturellement et instinctivement en orthographe phonétique. "

Si FB propose une réforme de l'orthographe basée sur le phonétisme, c'est qu'il est intéressé, et depuis longtemps, par les travaux de l'abbé Rousselot. En 1897, FB lui avait demandé d'intervenir dans les cours d'été de l'Alliance française, expérimenter la phonétique et rectifier des prononciations vicieuses. C'est alors une idée originale pour un universitaire: Enrica Galazzi, dans son article «Phonétique/Université/Enseignement des langues à la fin du $\mathrm{XIX}$ siècle », évoque les premiers pas de la phonétique de M. Rousselot et de P. Passy fondateur de l'Association Phonétique Internationale, ainsi la méfiance universitaire à leur encontre. Cela n'empêche pas FB d'aller «à Vienne en 1910 pour visiter un laboratoire de langues qui lui permettra de monter les Archives de la Parole en 1911 » (d'après J.-Cl. 
Chevalier, «F. Brunot, l'HLF et le français à l'étranger. Méthodes d'investigation », Langages, Larousse, juin 1994, p.61). Il y retournera en mission en 1912 pour y étudier « les archives des phonogrammes» comme l'indique l'arrêté du 25 avril 1912 signé par M. Guist'Hau, ministre de l'Instruction Publique et par M. Bayet Conseiller d'Etat.

Le 3 juin 1911, FB inaugure les Archives de la Parole, ancêtre de la Phonothèque Nationale devenue le département des documents sonores de la Bibliothèque nationale de France, et le 9 juin il est nommé le directeur de l'Institut de Phonétique de l'Université de Paris. C'est comme tel qu'il est désigné «pour représenter le Ministère de l'Instruction publique au premier Congrès International de Phonétique qui aura lieu à Hambourg du 19 au 22 avril 1914. » par un arrêté du 8 avril 1914. Les Archives de la Parole restent sous la direction de FB jusqu'en 1927. Hubert Pernot prend sa succession et de 1928 à 1938 quand les Archives de la Parole deviennent Musée de la Parole et du Geste en 1928. FB ne rompt ni avec l'Institut ni avec la maison Pathé qui a fourni le matériel pour ses enquêtes dialectologiques avec enregistrements, ni avec la phonétique. L'arrêté du 29 juin 1932 nomme encore FB membre du Conseil d'Administration de l'Institut de Phonétique. Cette science l'intéresse tellement que M. Roques, dans son discours du 26 novembre 1960 lu par le Doyen Aymard, lors de la célébration du centenaire de la naissance de FB, se souviendra encore de quelqu'un qui s'est attaché à la phonétique et qui a « constamment été préoccupé par la diversité des parlers de France, de leur unité et de la nécessité de les recueillir exactement, non seulement des parlers dialectaux, mais tous les parlers socialement distincts. »

Il évoque les enquêtes réalisées par FB dans les Ardennes et le Berry avec sa «roulotte d'enregistrement »: les langues parlées sont des langues vivantes, des assemblages de sons et peuvent constituer, grâce à la technologie nouvelle, une bibliothèque vivante qui servirait aux étudiants de langue ainsi qu'un musée de voix contemporaines.

Dans cette période de sa vie, FB construit son idéal de langue, faite avec de «beaux mots », avec une orthographe plus accessible à l'ensemble des citoyens et offre une nouvelle méthode d'étude, bouleversant ainsi un enseignement classique et ses valeurs culturelles - et sociales : «le français n'est plus la langue d'une bourgeoisie cultivée » - FB, note manuscrite au recto d'une feuille qui accompagne un texte manuscrit, Fonds Brunot à la Bibliothèque de l'Institut. FB restera toute sa vie fidèle à ses idées : en 1937, à 77 ans il propose comme sujet possible pour le $\mathrm{III}^{\mathrm{e}}$ Congrès des Professeurs de Langues Vivantes : «Comment la langue française est devenue nationale». Cette «nouvelle» langue il faut la diffuser par un enseignement moderne, il faut en faire la «propagande ». L'influence de la France passe par sa langue.

\section{La diffusion internationale du français}

Dès 1884 et jusqu'en 1924, il participe, dans le cadre de l'Alliance française, aux cours de vacances pour les élèves étrangers, dont il assume la première direction. En 1904, FB est «Président des cours permanents ». Ces cours sont destinés aux professeurs de français étrangers et sont sanctionnés par deux diplômes : le Diplôme d'aptitude à l'enseignement du français et le Diplôme d'assiduité qui deviennent le Diplôme Supérieur et le Diplôme Elémentaire. Ces cours sont enseignés en province, à Nancy en 1898, à Caen en 1899 et à Grenoble en 1900.

A ses débuts, de 1884 à 1914, au moment où FB s'y engage, l'enseignement dispensé par l'Alliance ne relève pas de la pure philanthropie : «son discours s'inscrit directement (...) dans la ligne du projet républicain de civisme et d'unification de la nation »., M. Roselli ( thèse, 1994, déjà citée) Le 16 février 1911, FB présente au Conseil le résultat de ses recherches concernant la question des cours permanents et propose « d'ouvrir des cours à toute personne, même française qui voudrait se consacrer à l'enseignement de notre langue au dehors et de placer ces cours sous le titre d'Ecole Supérieure de langue française » La proposition est adoptée à l'unanimité. C'est une intégration dans la filière de l'enseignement du français dans les pays étrangers. FB cumule ainsi des charges qui l'engagent à la fois dans la politique de l'Alliance et celle de l'Université. 
A l'étranger, il représente non seulement l'Université et l'étude scientifique de la langue mais aussi une forme d'adhésion politique à l'idéologie politique nationale - surtout à partir de 1922, année qui marque la reprise de ses déplacements. Alors Doyen de la Sorbonne (depuis 1919), professeur connu pour ses positions dans les réformes en cours, auteur très discuté de La Pensée et la Langue, etc. FB a acquis une stature internationale. Il fréquente tout autant les ministres - il est même familier avec certains - que les universitaires, à qui il propose ses services : «Je visiterai comme convenu l'Institut français et verrai l'ambassadeur qui doit présider ma première conférence. Si vous aviez quelque mission à remplir là-bas je suis à votre disposition. ", Lettre, 1925.

Il rend compte de ses missions. A son retour de Prague - du 13 au 29 avril 1923 - FB reprend dans une lettre de treize pages au Président du Conseil, Ministre des Affaires Etrangères, Raymond Poincaré (lettre transmise par voie hiérarchique par L. Bérard, son ministre de tutelle) : "Les idées que j'ai exposées sur la façon dont le français est devenu la langue diplomatique non sous la pression du roi de France mais par l'influence du génie français. »

\section{Son action diplomatique est appréciée :}

«Parmi les nombreuses conférences linguistiques qui sont données en Tchécoslovaquie et constituent un de nos meilleurs moyens de propagande, je dois signaler celles que vient de faire, sur l'invitation de l'Université Charles, M. Brunot, Doyen de la Faculté des Lettres de Paris parce que leur qualité et la personnalité du conférencier leur ont prêté une portée inhabituelle et ont été l'occasion de témoignages renouvelés de sympathie pour la France. »

Lettre de M F. Couget, Ministre de France à Prague, 25 mai 1925, à Son Excellence M. Poincaré, Président du Conseil et Ministre des Affaires Etrangères.

FB est donc souvent reçu à l'étranger comme un représentant officiel de la France : le Roi et la Reine de Belgique sont présents quand FB est reçu par l'Académie Royale à Bruxelles et c'est personnellement qu'il rencontre le Président tchèque : «J'ai été reçu par le Président Mazaryk avec lequel je suis resté plus de trois quart d'heure » (FB, lettre au Ministre des Affaires Etrangères).

Dans ses notes il précise :

$$
\begin{aligned}
& \text { «Il me reçoit à } 12 \mathrm{~h} 1 / 2 \text { sans me faire attendre. Apparence de vieillard (...) mais de } \\
& \text { vieillard robuste en pleine possession de sa force intellectuelle et morale (...) il } \\
& \text { affirme que le français est la première langue qu'il ait apprise (...) ce choix (Romain } \\
& \text { Rolland et Péguy) m'étonne et je m'abstiens de toute réflexion mais c'est un indice } \\
& \text { qu'il étudie le français à travers les livres allemands. L'entretien a duré trois quarts } \\
& \text { d'heure et c'est moi qui ai mis fin.» }
\end{aligned}
$$

En Suède, FB est étonné de ne pas avoir été reçu par le roi de Suède, Haakon, malgré la chaleur de la réception...

FB effectue de nombreux voyages à l'étranger : Suisse, Belgique, Angleterre, Tchécoslovaquie, Bohême, Autriche, Allemagne, Pologne, Roumanie, Hollande, Norvège, Danemark et Suède. Il y donne des cours et des conférences où il mêle habilement l'étude de la langue à ses vues personnelles car il faut donner « une impression de science à la française », ajoutant «j'ai essayé de voir dans l'évolution même de la pensée et de la vie française », ce qui signifie qu'il faut présenter la langue française sous ses aspects

- politique : comment le français est devenu l'instrument de la diplomatie internationale,

- sociologique : comment la démocratie contemporaine,

- littéraire : comment le français a été renouvelé par V. Hugo,

- philologique: de la nécessité d'une classification nouvelles des faits linguistiques. (référence à $L a$ Pensée et la langue).

Pour soutenir cette politique de propagande il est nécessaire de choisir ses sujets : 
«Il ne faut pas faire connaître de notre littérature française les pages les plus scabreuses mais les chefs d'œuvre de l'art, de l'esprit, la France épique des grandes luttes, la France des énergies constructrices dans son œuvre de civilisation en Europe et hors d'Europe, c'est là sa tâche... »

Les pays francophones sont aussi concernés par cette propagande. Reçu en Suisse, à la Chaux-de-Fonds, FB donne une conférence intitulée, sans ambiguïté « Du Français de France ».

FB milite ainsi par ses conférences pour la langue française mais cela lui semble insuffisant. Aussi s'active-t-il pour être soutenu en permanence par un enseignement efficace. Il assure un suivi de l'Alliance Française et des instituts français implantés à l'étranger et en rend compte au ministère de l'Instruction publique - ou plutôt à ses ministres. Il organise ainsi un enseignement de la langue française à l'étranger en disposant de moyens d'ordre politique, administratif, financier. Il facilite la circulation non seulement des professeurs mais aussi des étudiants : des bourses sont créées, les diplômes sont valorisés et le contrôle facilité. Sa politique commence à prendre :

\begin{abstract}
«Nous avons eu une conversation toute particulière après le repas. Il serait favorable à des propositions d'échanges réguliers des professeurs. » (FB à Berlin)

«Je crois que le plus important de notre rôle est de faciliter les relations universitaires entre nos deux pays et de préparer autant que possible des étudiants tchécoslovaques à visiter les universités françaises ». (Lettre de M. Tibal, professeur à l'Université de Prague, à FB, 1923)
\end{abstract}

FB, qui surveille de près les relations entre les universités et l'Alliance Française, perçoit clairement les faiblesses et les manques dans les structures qu'il contribue à mettre en place. Il faudrait au moins alimenter régulièrement les bibliothèques et favoriser des lancements de programme, comme celui de la phonétique à l'Université de Prague: "L'Université (de Prague) pourrait enregistrer les dialectes slovaques. Elle achèterait volontiers une machine de voyage comme la nôtre ». De plus, il insiste pour qu'on améliore les ressources financières des professeurs mais aussi qu'on assure la qualité de l'enseignement afin d' " éloigner les médiocres ».

\title{
4 Un foyer pour les étudiants étrangers à Paris
}

FB n'a guère confiance en la valeur des diplômes de français donnés à l'étranger. Bien sûr les étudiants peuvent venir en France, mais il en vient peu, par exemple à Caen, Grenoble et Nancy où ils ne se sentent pas forcément bien intégrés et où par ailleurs ils échappent à la tutelle, même indirecte, de FB. Il faut donc instaurer un modèle français à Paris :

« En principe on admet à Leipzig comme à Vienne, les Universités comme le Ministre de l'Instruction Publique d'Autriche, que les étudiants doivent avoir un heim à Paris. C'est l'argent qui est à trouver. J'ai fait sentir que l'on pouvait retenir un terrain ...» (FB à Vienne)

De quel terrain s'agit-il ? Le même que le Recteur Paul Appell désigne, lors d'une promenade, à M. Deutsch de la Meurthe suite à une conversation, au début du mois de mai 1920 : il s'agit d'un terrain militaire désaffecté, compris dans les fortifications, situé Boulevard Jourdan. Pourquoi ce choix ? L'emplacement est situé à proximité de la Sorbonne, il est mitoyen du quartier des Ecoles, il est relativement facile d'obtenir ces terrains militaires désaffectés et c'est l'arrondissement le plus sain de Paris.

Le Recteur dans la conversation évoque la situation matérielle des étudiants avec M. Deutsch qui se montre disposé à créer, à côté de l'Université dispensatrice de savoir, «un abri lumineux, aéré, confortable et calme (...) où la jeunesse des Facultés et des grandes Ecoles pût poursuivre en paix ses études et parachever sa formation intellectuelle. » Et le 17 mai 1920, M Deutsch offre à l'Université de Paris dix millions de francs-or pour loger 350 étudiants. Le 24 juin 1920, P. Appell remercie et 
communique sa lettre à A. Honnorat en lui demandant d'obtenir la concession du terrain auprès du Ministre de la Guerre. La réponse est négative, comme déjà en 1919, mais

« Le ménage Brunot avait pour ami le Directeur du Génie au ministère de la Guerre dont ce terrain dépendait dans toute la zône aedificandi (...) entre temps aussi, le député soldat de deuxième classe Honnorat lance pour la première fois l'idée d'une cité universitaire à Paris et l'expose à un congrès tenu à Londres en septembre 1918 (...) mais il devient Ministre de l'Instruction publique et déjeune chez ses amis Brunot ... ».

Ces propos sont confirmés par André Brunot sur une note biographique qu'il a écrite sur son père (p. 7 et p. 11 de ma thèse) :

« La Sorbonne ne put procéder à ces réalisations (constructions de locaux) que grâce à des recours privés. A celui de la «marquise » s'ajouta celui de Deutsch de la Meurthe, qui permit la création de la Cité Universitaire qui doit à $\mathrm{FB}$ au moins son implantation dans le $14^{\mathrm{ème}}$ (...) F. Brunot parvenait à ces résultats grâce à ses bons rapports qu'il possédait avec les ministres successifs : Honnorat et A. de Monzie en particulier. »

Le 16 juillet 1920 une entrevue a lieu associant A. Honnorat, le Directeur de l'Enseignement Supérieur, le Préfet de la Seine, Henri Goldet et Henri Becker qui représente M Deutsch de la Meurthe. L'Etat, pour en doter l'Université, consent à se faire rétrocéder un lot de terrains, les trois bastions $(81,82,83)$ compris entre la rue de la Tombe Issoire et la Porte de Gentilly. Après la Convention du 7 juin 1921 entre la Ville et l'université la loi du 28 juin 1921 alloue à l'Université 15 unités de neuf cents mille francs.

Le décret du 6 juin 1925 reconnaît la Fondation nationale de la Cité Universitaire d’Utilité Publique

Après son inauguration la Cité Universitaire continue de s'agrandir. Le 11 juin 1927 : donation par David Weill de deux millions à l'Université pour racheter à la Ville la moitié des terrains de l'ancienne enceinte. La loi du 20 juillet 1928 exproprie 4ha à la Commune de Gentilly, etc. On peut supposer que les liens entre A. Honnorat et FB ont joué un rôle décisif. Les deux hommes sont amis et entretiennent autour de la cité Universitaire des relations amicales tout au long des années :

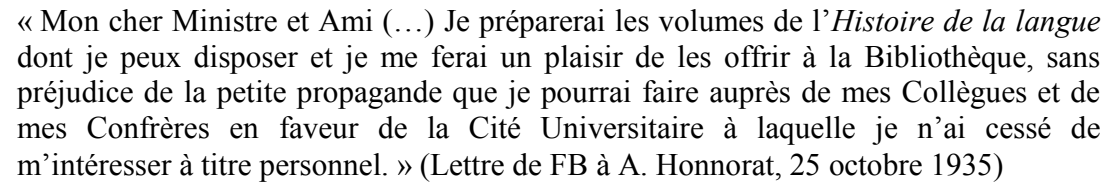

Le rôle de FB parait modeste mais essentiel. Déjà, sans lui, le ministère de la guerre aurait probablement conservé ses fortifications encore un certain temps - pour ne pas dire un temps certain. FB qui avait été Maire du XIV ${ }^{\mathrm{e}}$ arrondissement de Paris entre 1911 et 1919 a conservé en 1920 ses projets et peut-être pas encore digéré le premier refus du Ministre de la Guerre. FB dès le premier instant du projet et de sa réalisation fait partie intégrante de la gestion de la Cité universitaire.

«En tant que maire FB nourrissait des projets d'urbanisme, notamment celui d'une grande voie reliant les jardins de l'Observatoire au parc de Montsouris. S'il ne vit se réaliser, grâce à André Honnorat, que celui de la Cité universitaire, tout porte à croire que la responsabilité de la Commission lui a été confiée en considération de cet intérêt particulier. », Gérard Chevalier, sociologue, chercheur au CNRS.

Le 7 mars 1921 est créée la Commission du Conseil de l'Université de Paris, restreinte à partir du 28 mai 1923 - toujours en présence de FB qui, évidemment fait partie du Conseil d'administration de la Cité Universitaire créée le 5 décembre 1921 par le Conseil de l’Université.

La réalisation de la Cité Universitaire Internationale est la dernière pièce du puzzle construit par FB sa vie durant avec une conviction sans faille pour illustrer son idéal de langue française et unifier la nation mais aussi pour propager cette langue purifiée hors de ses frontières et consolider le prestige de la France : 
ISBN 978-2-7598-0358-3, Paris, 2008, Institut de Linguistique Française

Ouverture

DOI $10.1051 / \mathrm{cmlf0} 8355$

« Il y a longtemps que ce prestige n'avait été aussi grand. Le génie de la race nous fait regagner d'un côté le terrain que les causes économiques et politiques nous enlèvent d'un autre. Et si une part de cette belle victoire, la plus grande, est due aux lettres françaises, une autre appartient au monde enseignant. » (FB, Revue Hebdomadaire, 14 juillet 1911)

\section{Repères bibliographiques}

Brunot Ferdinand, (1936) La Pensée et la langue, Masson et $C^{\text {ie }}$ Editeurs

Bibliothèque de l'Institut :

Fonds Brunot

Spont Henry, (1932) L'université de Paris et la Cité Universitaire, Paris, Lang.

Bourrelier, (1936) La vie au quartier latin, Paris

Aux Archives nationales

Dossier F/17/24348

Thèses

Roselli Mariangela, (1994) La langue française entre science et République, 1880-1950

Anne Coste, (1996) L'œuvre grammaticale de Ferdinand Brunot à travers les archives de l'Institut 